\title{
Fixed Point Theorems with Applications to the Solvability of Operator Equations and Inclusions on Function Spaces
}

\author{
Nawab Hussain, ${ }^{1}$ Jesus Garcia-Falset, ${ }^{2}$ Mohamed-Aziz Taoudi, ${ }^{3,4}$ and Calogero Vetro ${ }^{5}$ \\ ${ }^{1}$ Department of Mathematics, King Abdulaziz University, P.O. Box 80203, Jeddah 21589, Saudi Arabia \\ ${ }^{2}$ Department of Mathematical Analysis, University of Valencia, Spain \\ ${ }^{3}$ Centre Universitaire Polydisciplinaire, Kelaa des Sraghna, Morocco \\ ${ }^{4}$ Université Cadi Ayyad, Laboratoire de Mathématiques et de Dynamique de Populations, Marrakech, Morocco \\ ${ }^{5}$ Department of Mathematics and Computer Science, University of Palermo, Via Archirafi 34, 90123 Palermo, Italy
}

Correspondence should be addressed to Nawab Hussain; nhusain@kau.edu.sa

Received 14 April 2015; Accepted 14 April 2015

Copyright (C) 2015 Nawab Hussain et al. This is an open access article distributed under the Creative Commons Attribution License, which permits unrestricted use, distribution, and reproduction in any medium, provided the original work is properly cited.

Fixed point theory is an elegant mathematical theory which is a beautiful mixture of analysis, topology, and geometry. It is an interdisciplinary theory which provides powerful tools for the solvability of central problems in many areas of current interest in mathematics and other quantitative sciences, such as physics, engineering, biology, and economy. In fact, the existence of linear and nonlinear problems is frequently transformed into fixed point problems, for example, the existence of solutions to partial differential equations, the existence of solutions to integral equations, and the existence of periodic orbits in dynamical systems. This makes fixed point theory a topical area and a subject of active scientific research, constantly evolving and growing and in a perpetual progress.

Fixed point theory has two main branches: on one hand, we can consider the results obtained by using metric properties; on the other hand, we can consider the results deduced from topological properties. Among the classical results, which are the basis for the metric branch, we retain the Banach contraction principle in complete metric spaces established by Banach in 1922. With respect to the topology branch, the main theorems are those of Brouwer and his infinite dimensional version, the Schauder fixed point theorem, which were proved in 1912 and 1930, respectively. In both, the compactness plays an essential role. A hybrid result combining metric and topological properties was established by Krasnoselskii in 1955. This result is exciting and has a very wide scope. In recent years, a number of authors have found this theorem a very satisfactory outcome for the study of stability and asymptotic behavior of solutions for certain differential equations which resisted the direct Lyapunov method.

The development of such fixed point theorems remains a constant concern for many investigators who have continued to enrich this field by new quite interesting contributions following constantly the progress of applications to fully meet the needs of this dynamic and active field. Thus, fixed point theorems were developed for single-valued and multivalued mappings on topological vector spaces, metric spaces, Banach spaces, Banach algebras, posets, lattices, Banach lattices, and so forth.

Due to the importance of fixed point theory and its applications, it is worthwhile to publish a special issue on this topic to highlight recent advances made by mathematicians actively working in this area.

This special issue was originally elaborated to report the latest advances in fixed point theory in abstract spaces and their applications. It includes works on single-valued and multivalued mappings in normed and metric spaces and various applications to boundary value problems, equilibrium problems, and variational inequalities.

Nawab Hussain Jesus Garcia-Falset Mohamed-Aziz Taoudi Calogero Vetro 


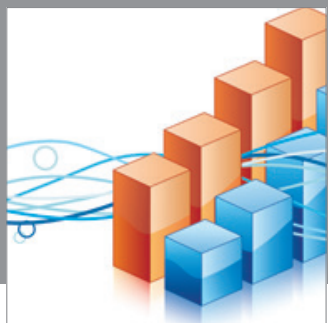

Advances in

Operations Research

mansans

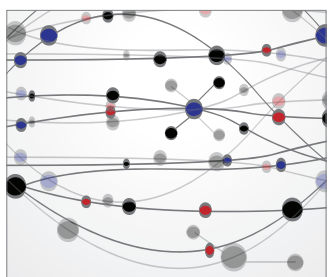

The Scientific World Journal
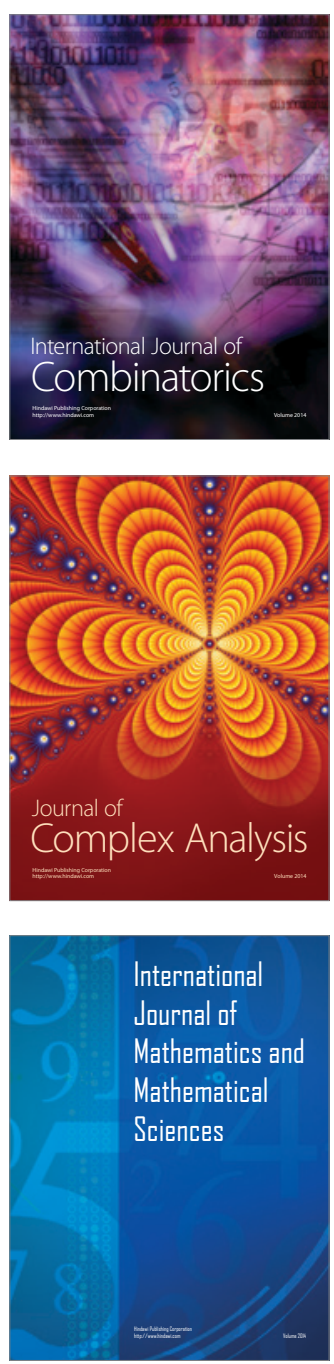
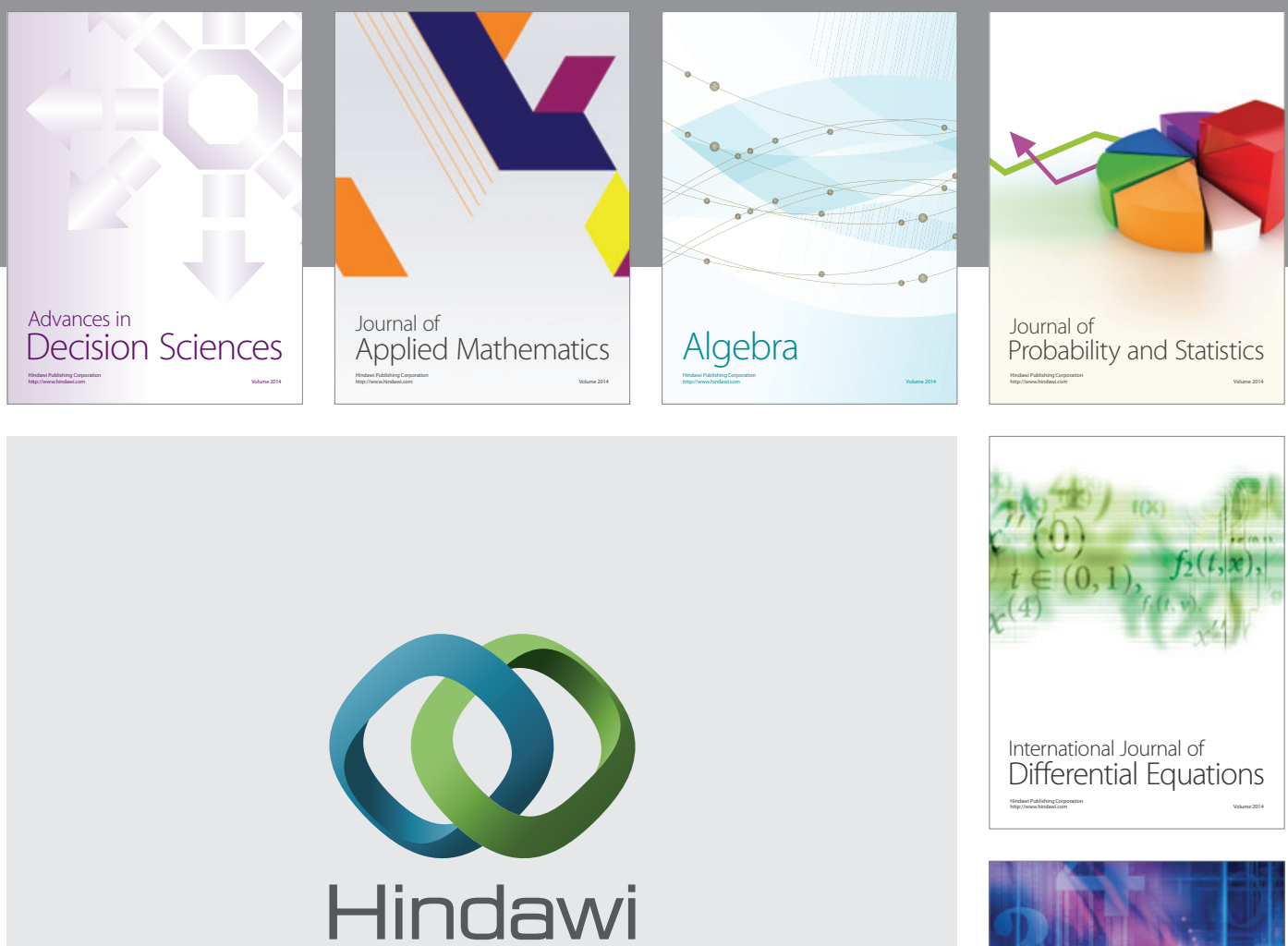

Submit your manuscripts at http://www.hindawi.com
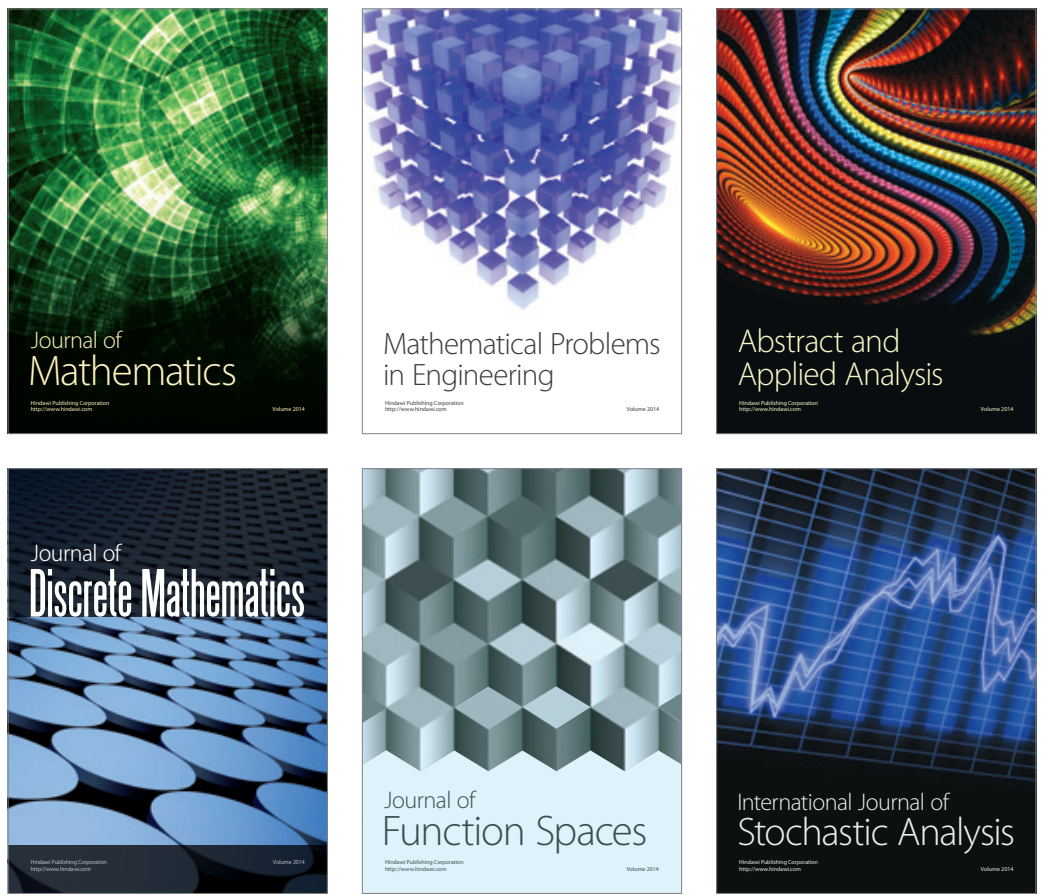

Journal of

Function Spaces

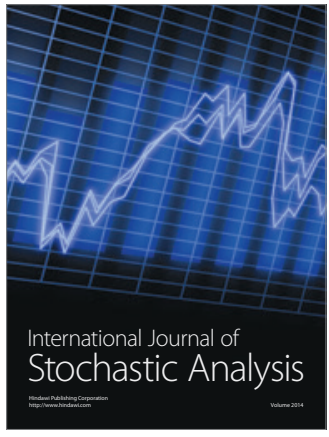

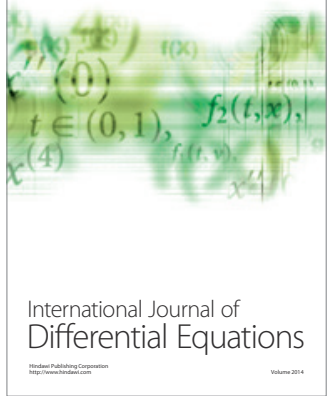
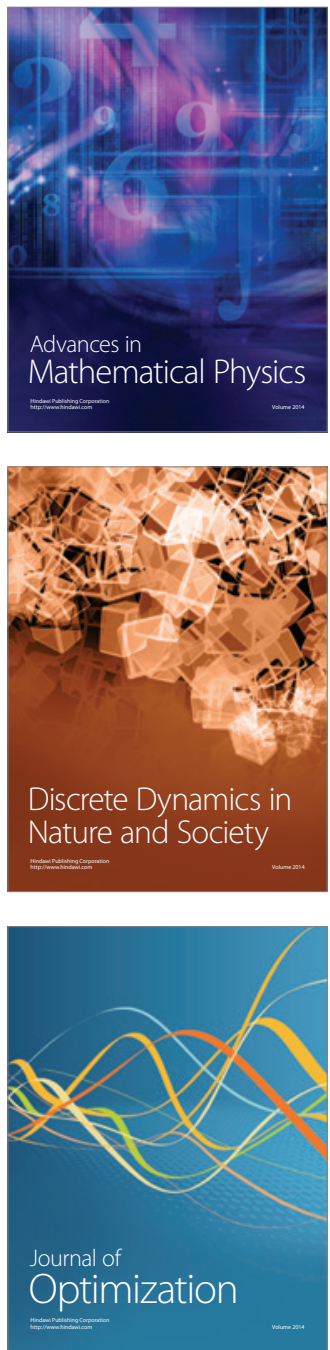